\title{
The Myristoylated Protein Rapsyn is Cotargeted with the Nicotinic Acetylcholine Receptor to the Postsynaptic Membrane via the Exocytic Pathway
}

\author{
Sophie Marchand, Fabrizia Bignami, Françoise Stetzkowski-Marden, and Jean Cartaud \\ Biologie Cellulaire des Membranes, Département de Biologie Supramoléculaire et Cellulaire, Institut Jacques Monod, \\ Unité Mixte Recherche 7592, Centre National de la Recherche Scientifique, Universités Paris 6 et Paris 7, 75251, Paris \\ Cedex 05, France
}

Rapsyn, a $43 \mathrm{kDa}$ protein required to cluster nicotinic acetylcholine receptors (AChRs) at the neuromuscular junction, is tightly associated with the postsynaptic membrane via an $\mathrm{N}$-terminal myristoylated site. Recent studies have shown that some acylated proteins associate with the exocytic pathway to become targeted to their correct destination. In this work, we used Torpedo electrocyte to investigate the intracellular routing of rapsyn compared to those of AChR and Na,K-ATPase, the respective components of the innervated and noninnervated membranes. We previously demonstrated that these latter two proteins are sorted and targeted to plasma membrane via distinct populations of post-Golgi vesicles (Camus et al., 1998).

Rapid and efficient synaptic transmission at the neuromuscular junction is provided by the accumulation of nicotinic acetylcholine receptors (nAChRs) at postsynaptic sites directly across the presynaptic area of neurotransmitter release. The accumulation and maintenance of high concentrations of AChRs at newly formed synaptic sites of the neuromuscular junction involve several levels of regulatory mechanisms, including transcriptional regulation of the genes encoding the AChR subunits, clustering, and stabilization of AChR via the cytoskeleton (for review, see Sanes and Lichtman, 1999). The demonstration of a subneural sarcoplasm housing a specialized Golgi apparatus (GA) (Jasmin et al., 1989, 1995; Antony et al., 1995) and a local network of stable microtubules (Jasmin et al., 1990) thereby illustrates the compartmentalization of the exocytic pathway in innervated muscle fibers and its specialization in the synaptic region (for review, see Cartaud and Changeux, 1993). This implies that additional post-translational regulatory mechanisms involving local synthesis, sorting, and targeting must exist to ensure the proper supply of AChRs at synaptic sites.

Sorting and targeting neosynthetized membrane proteins in the secretory pathway participate in the genesis and maintenance of specialized domains of the cell surface (Griffiths and Simons, 1986; Wandinger-Ness et al., 1990). In a recent work, we studied

Received June 21, 1999; revised Sept. 24, 1999; accepted Oct. 21, 1999.

This work was supported by the Centre National de la Recherche Scientifique, The Universities Paris 6 and Paris 7, and the Association Française contre les Myopathies. F.B. is a recipient of a postdoctoral grant from the Fondation Institut Pasteur/Fondazione Cenci-Bolognetti. We thank Drs. S. C. Froehner and S. Tzartos for generous gifts of antibodies.

Correspondence should be addressed to Jean Cartaud, Institut Jacques Monod, Université Paris 7, Tour 43, 2 Place Jussieu 75251, Paris Cedex 05, France. E-mail: cartaud@ijm.jussieu.fr.

Copyright (C) 2000 Society for Neuroscience $\quad 0270-6474 / 00 / 200521-08 \$ 15.00 / 0$
Biochemical and immunoelectron microscopy analyses of various populations of post-Golgi vesicles immunopurified with magnetic beads led us to identify post-Golgi transport vesicles containing both rapsyn and AChR. These data suggest that rapsyn, as for $A C h R$, specifically follows the exocytic pathway. Furthermore, immunogold-labeling experiments provided in situ evidence that $\mathrm{AChR}$ and rapsyn are cotransported in the same post-Golgi vesicles. Taken together, our observations suggest that rapsyn and AChR are cotargeted to the postsynaptic membrane.

Key words: rapsyn; myristoylation; nicotinic acetylcholine receptor; targeting; synapse; Torpedo electrocyte

the targeting of AChR to the postsynaptic membrane in Torpedo electrocyte. In this study, two subpopulations of post-Golgi vesicles (PGVs) enriched either in AChR or in Na,K-ATPase, the two major components of the innervated and noninnervated membrane domains, have been characterized suggesting an efficient intra-Golgi sorting of these two proteins and a direct targeting of AChR to the postsynaptic membrane via post-Golgi vesicular transporters (Camus et al., 1998).

The accumulation of AChR in the postsynaptic membrane is a complex process involving integral, cytoskeletal, and extracellular matrix components. Among the proteins of the postsynaptic cytoskeleton, the myristoylated $43 \mathrm{kDa}$ protein, rapsyn, is of particular interest. Knock-out mice and cotransfection experiments have demonstrated that rapsyn is required for AChR clustering (Froehner et al., 1990; Phillips et al., 1991a; Yu and Hall, 1994; Gautam et al., 1995). As for other myristoylated proteins (Wilcox et al., 1987), rapsyn is myristoylated on its N-terminal Gly during translation (Musil et al., 1988). This modification confers membrane targeting to rapsyn (Phillips et al., 1991b; Ramarao and Cohen, 1998). Two possibilities can then be envisaged: as generally proposed, rapsyn could insert directly into the plasma membrane after synthesis. Alternatively, as shown for other acylated proteins (Liu et al., 1994; Gonzalo and Linder, 1998; Bijlmakers and Marsh, 1999), rapsyn might initially be targeted to intracellular membranes of the exocytic pathway and subsequently travel to the cell surface.

In this work, we have investigated the intracellular routing of rapsyn as compared to that of AChR or Na,K-ATPase in Torpedo electrocyte. Our data bring evidence that rapsyn associates to distal exocytic compartments (post-Golgi vesicles) and is specifically cotargeted with AChR to the postsynaptic membrane. 


\section{MATERIALS AND METHODS}

Biological material. Torpedo marmorata were obtained from the Station de Biologie Marine, Roscoff (Université Paris 6) and the Station de Biologie Marine, Arcachon (Université Bordeaux I), France.

Antibodies. The following antibodies were used for immunopurification, immunoblotting, and immunocytochemistry: guinea pig polyclonal anti-AChR and rabbit anti-Na,K-ATPase antibodies directed respectively against Torpedo AChR and Torpedo Na,K-ATPase pump have been previously characterized (Camus et al., 1998). Rat monoclonal antibodies directed against cytoplasmic epitopes of AChR $\alpha$-subunit (mAbs 111 and 155) were kindly provided by Dr. S. Tzartos (Tzartos and Lindstrom, 1980). Monoclonal antibody directed against rapsyn (mAb 1234A) was generously provided by Dr. S. Froehner (Peng and Froehner, 1985). Monoclonal anti-phosphoserine antibodies were obtained respectively from Upstate Biotechnology (Lake Placid, NY) and Sigma (St. Louis, MO). Biotinylated goat anti-rabbit, goat anti-mouse, and sheep anti-rat antibodies were purchased from Amersham.

Purification of AChR-rich plasma membranes and PGVs. AChR-rich plasma membranes from fresh electric tissue were prepared according to Saitoh and Changeux (1980). Crude PGVs were isolated following a protocol adapted from Wandinger-Ness et al. (1990) for the purification of PGVs in Madin-Darby canine kidney cells (Camus et al., 1998). Antiproteases ( $1 \mathrm{~mm}$ leupeptin, $1 \mathrm{~mm}$ pepstatin, $0.2 \mathrm{~mm}$ PMSF, $0.5 \mathrm{mg} / \mathrm{ml}$ aprotinin, and $0.8 \mathrm{~mm}$ benzamidin) were added throughout the purification procedures. Crude PGVs and Golgi fractions were stored in liquid nitrogen to await further investigation. To avoid a possible contamination of crude PGVs or Golgi fractions by soluble rapsyn, which is present in small quantity in the cytoplasm of the electrocytes, the fractions were washed and pelleted by centrifugation at $250,000 \times g$ for $1 \mathrm{hr}$ before biochemical analyses.

Immunoisolation of post-Golgi vesicles. PGVs fractions were further immunopurified according to their protein content using magnetic beads, as described in Camus et al. (1998). To improve the efficiency of the immunoabsorbants, biotinylated linker antibodies were used to fix antiNa,K-ATPase, mAbs 111 and 155 anti- $\alpha$ AChR subunit or mAb 1234A anti-rapsyn antibodies to $2.8 \mu \mathrm{m}$ diameter streptavidin-conjugated magnetic beads (Dynabeads M-280; Dynal AS, Oslo, Norway) according to manufacturer's instructions (typically $20 \mu \mathrm{g}$ of $\mathrm{IgGs} / \mathrm{mg}$ of beads). Immunopurification of PGVs was performed by incubation overnight at $4{ }^{\circ} \mathrm{C}$ of the crude PGV fraction (400 $\mu \mathrm{l} / \mathrm{mg}$ of beads) in PGV buffer $(1.2 \mathrm{~mm}$ phosphate buffer, $\mathrm{pH} 7.4$, containing (in $\mathrm{mM}$ ): $280 \mathrm{KCl}, 3 \mathrm{NaCl}, 3.4$ $\mathrm{MgCl}_{2}, 1.8 \mathrm{CaCl}_{2}, 100$ sucrose, and 5 glucose) supplemented with 5\% BSA in PBS and antiproteases to $1 \mathrm{ml}$ final incubation volume. For biochemical analysis by SDS-PAGE and Western blotting, beads were directly resuspended in Laemmli buffer (Laemmli, 1970).

Cross-linking experiments. Cross-linking experiments were performed according to the protocol described by Burden et al. (1983) using a heterobifunctional cross-linking reagent, succinimidyl 4-( $p$-maleimidophenyl)butyrate (SMPB), that contains $N$-ethylmaleimide and $N$-hydroxysuccinimide as reactive groups $(0.12 \mathrm{~nm}$ between reactive groups). Briefly, purified AChR-rich membranes, crude plasma membranes, and crude PGVs were washed with $10 \mathrm{~mm}$ sodium phosphate buffer, $1 \mathrm{~mm}$ EDTA, $1 \mathrm{~mm}$ EGTA, $0.3 \mathrm{~mm}$ PMSF, and $0.02 \%$ sodium azide, $\mathrm{pH} 7.4$, pelleted by centrifugation and resuspended in $10 \mathrm{~mm}$ sodium phosphate buffer and $1 \mathrm{~mm}$ EDTA, $\mathrm{pH} 8.0$, at a final concentration of $4 \mathrm{mg}$ of protein $/ \mathrm{ml}$. SMPB in DMSO ( $2 \% \mathrm{v} / \mathrm{v}$ stock solution) was added to the membranes at concentrations of $10^{-7}$ to $10^{-4} \mathrm{M}$ and incubated for $30 \mathrm{~min}$ at room temperature in the dark. Membranes were then pelleted and washed in $10 \mathrm{~mm}$ sodium phosphate buffer, $1 \mathrm{~mm}$ EDTA, pH 8, before solubilization in SDS-PAGE sample buffer. Forty micrograms of membrane proteins were loaded in each lane. Analysis of the cross-linked products were subsequently performed by Western blotting followed by ECL detection.

SDS-PAGE and Western blotting. Proteins from the various membrane preparations were separated on 8 or $10 \%$ SDS-PAGE in a slab cell (Mini protean II; Bio-Rad, Richmond, CA). In all experiments, heating of the sample buffer was omitted to prevent aggregation of $\mathrm{Na}, \mathrm{K}$-ATPase and dephosphorylation of the AChR. After separation, proteins were electrotransfered onto nitrocellulose paper (Schleicher \& Schuell, Dassel, Germany) according to Towbin et al. (1979). Immunoblot experiments were performed as described elsewhere (Cartaud et al., 1993). For phosphoserine detection, skimmed milk in the blocking solutions was replaced by $3 \%$ gelatin. Detection of the signal was achieved with a chemiluminescent reaction (ECL; Amersham) using x-ray films (Fuji Photo Film Company, Tokyo, Japan). Antibody dilutions were 1:3000 for anti- $\alpha$ AChR subunit, 1:10,000 for anti-rapsyn, 1:40,000 for anti-Na,KATPase, and 1:10,000 for anti-phosphoserine.

Electron microscopy and immunocytochemistry. For immunolabeling, columns of electrocytes were dissected and immediately incubated at room temperature in a microtubule-stabilizing buffer $[100 \mathrm{~mm}(N-$ morpholino)-ethanosulfonic acid, $\mathrm{pH}$ 6.4, 1 mM EGTA, 1 mM GTP, 0.5 $\mathrm{mm} \mathrm{MgCl} 2$, and $10 \mu \mathrm{M}$ taxol; Schiff et al., 1979] for $60 \mathrm{~min}$. Subsequently, electric tissue was fixed with $3 \%$ paraformaldehyde and $0,05 \%$ glutaraldehyde in $0.1 \mathrm{M}$ phosphate buffer, $\mathrm{pH} 7.4$, at $4^{\circ} \mathrm{C}$, impregnated with $25 \%$ sucrose (w/v) and rapidly frozen in melting Freon R-22 cooled by liquid nitrogen. Frozen sections $(4 \mu \mathrm{m})$ were obtained by cutting the columns in a cryostat (SLEE, London, UK) at $-24^{\circ} \mathrm{C}$. For optimal visualization of cell polarity, transversally oriented sections of electric tissue were selected. Sections were recovered onto ovalbumine-coated glass slides, air-dried, and stored at $-70^{\circ} \mathrm{C}$ until further processing.

Immunogold experiments on electric tissue were performed using a pre-embedding labeling technique (Kordeli et al., 1986; Camus et al., 1998). After preincubation in PBS containing 4\% BSA and $1 \%$ fish gelatin, cryostat sections were incubated for $1 \mathrm{hr}$ at room temperature with the first antibody (anti- $\alpha$ AChR subunit, mAbs 111 and 155, 1:100 and/or anti-rapsyn 1:200) in PBS containing $0.4 \%$ BSA and $0.1 \%$ fish gelatin. After thorough washing, sections were incubated with goat anti-rat IgGs conjugated to $6 \mathrm{~nm}$ colloidal gold particles and/or goat anti-mouse IgGs conjugated to $12 \mathrm{~nm}$ colloidal gold particles (AuroProbe EM; Amersham). In these experimental conditions, a very low background signal was observed with secondary antibodies alone. Subsequently, sections were fixed for $45 \mathrm{~min}$ with $2.5 \%$ glutaraldehyde in $0.1 \mathrm{M}$ cacodylate buffer, $\mathrm{pH} 7.4$, containing $0.1 \%$ tannic acid, post-fixed for 45 min with $1 \%$ osmium tetroxide in $0.1 \mathrm{M}$ cacodylate buffer, dehydrated in a series of ethanol solutions, and embedded in epoxy resin. Serial thin sections (silver to gold) were directly observed in the EM without further staining. For EM immunodetection of antigens in PGVs, Dynabeads were processed as cryosections, pelleted, fixed, and embedded. Thin sections were stained with 5\% uranyl acetate and $1 \%$ lead citrate. All sections were observed with a Philips CM12 electron microscope operating at 60 or $80 \mathrm{keV}$. Micrographs were taken on Kodak (Eastman Kodak, Rochester, NY) EM 4489 electron microscope films.

\section{RESULTS \\ Rapsyn is specifically associated with AChR-containing PGVs}

In a previous study, we have shown that AChR and Na,K-ATPase are associated respectively with discrete membrane compartments of the exocytic pathway (PGVs) of the electrocyte. In a first attempt to unravel the targeting of rapsyn, we looked for its presence in the exocytic pathway. We isolated crude PGVs and crude Golgi using sucrose equilibrium gradient centrifugation (Camus et al., 1998). PGVs with a buoyant density of 1.08-1.10, a mean diameter of $80-100 \mathrm{~nm}$, and Golgi membranes (buoyant density of 1.11-1.15) were collected from the same gradient. The respective content in rapsyn and $\mathrm{AChR}$ of the crude vesicles as well as that of the crude Golgi fraction were analyzed by Western blotting after SDS-PAGE and compared to that of AChR-rich plasma membranes. As seen in Figure 1, rapsyn was immunodetected both in GA and PGVs. In the Torpedo postsynaptic membrane, equal amounts of AChR $\alpha$-subunit and rapsyn have been detected (LaRochelle and Froehner, 1986). In our experimental conditions, rapsyn detection was more efficient than that of AChR by a factor of 2 or 3 (Fig. 1, legend). In the Golgi fraction, rapsyn was usually detected at a very low level (Fig. 1), whereas crude $\mathrm{PGVs}$ disclose rapsyn/AChR ratio close to that found in postsynaptic membranes. These observations suggest that rapsyn is associated with intracellular compartments of the exocytic pathway.

As previously shown, AChR and Na,K-ATPase are present in distinct populations of PGVs, supporting the notion that these two proteins are intracellularly sorted within the trans-Golgi network (Camus et al., 1998). As a consequence, it is conceivable that if rapsyn is associated with the secretory pathway, it will take 


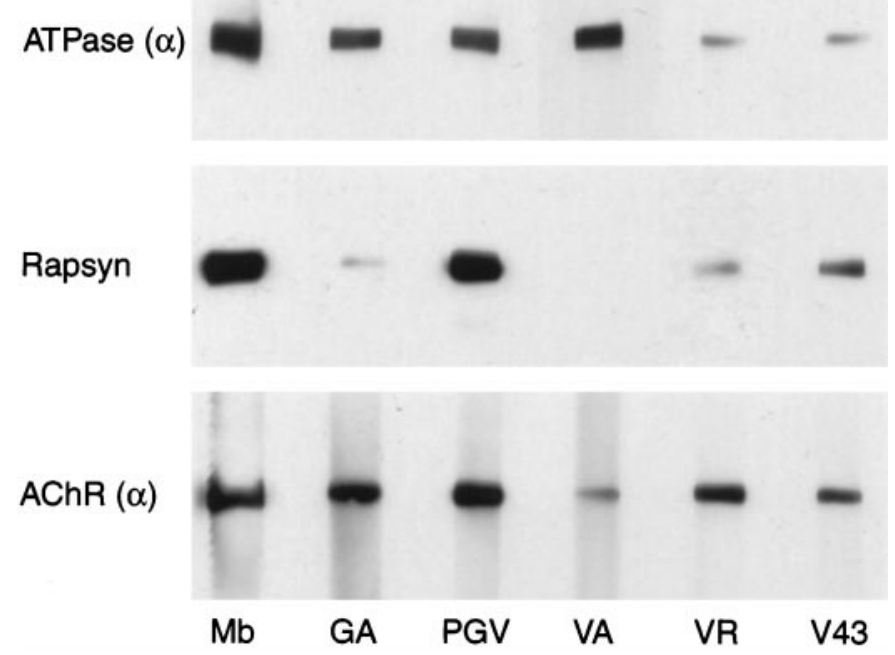

Figure 1. Western blot detection of rapsyn, AChR, and Na,K-ATPase in exocytic compartments of Torpedo electrocyte. The protein content of the Golgi fraction $(G A)$, crude PGVs, and immunopurified PGV fractions (VA VR, and V43) was analyzed by immunoblotting after SDS-PAGE using polyclonal anti-Na,K-ATPase and anti-AChR antibodies, and $\mathrm{mAb}$ 1234 anti-rapsyn, and compared to Torpedo postsynaptic membranes $(\mathrm{Mb})$. The relative intensities of the AChR ( $\alpha$-subunit) and $\mathrm{Na}$, K-ATPase ( $\alpha$-subunit) bands were roughly equivalent in postsynaptic membranes, Golgi, and crude PGV fractions. Compared with the crude PGV fraction, VA and VR were enriched in Na,K-ATPase and AChR, respectively (Camus et al., 1998). Immunodetection of rapsyn in postsynaptic membrane fraction appears more efficient than AChR immunodetection by a factor 2 or 3, taking into account the 1:1 stoichiometry between these two molecules (LaRochelle and Froehner, 1986). Rapsyn/ AChR ratio was equivalent in postsynaptic membranes and crude PGV fractions. A much lower amount of rapsyn was detected in the Golgi fraction. Rapsyn was absent from VA fraction. VR contained variable but always low amounts of rapsyn, whereas V43 displayed amounts of rapsyn ranging from 30 to $50 \%$ of $\mathrm{AChR}$.

a specific route to the postsynaptic membrane. To test this hypothesis, distinct populations of PGVs were purified, using immunomagnetic beads, on the basis of their content in AChR, rapsyn, and Na,K-ATPase. The protein content of each population was determined using Western blotting experiments and taking into account the rapsyn/AChR ratio established for the postsynaptic membranes fraction (Fig. 1, legend). As previously shown by Camus et al. (1998), PGVs isolated with anti-Na,KATPase (VA) and with anti-AChR (VR) were enriched in Na,KATPase and AChR, respectively (Fig. 1). Rapsyn was only detected in VR, although in low and variable amounts. VA were devoid of rapsyn (Fig. 1). These data suggested a cotransport of rapsyn and $\mathrm{AChR}$ in the same post-Golgi vesicles. To confirm this cotransport, we isolated a population of PGVs using magnetic beads coated with anti-rapsyn antibodies (V43). Western blotting analysis revealed that these PGVs contained large, although generally lower, amount (30-50\%) of rapsyn compared to AChR $\alpha$-subunit, as compared to postsynaptic membranes and crude PGV fraction (Fig. 1). Interestingly, only traces of Na,K-ATPase were detected in V43, this finding strengthening our current hypothesis of a separate intracellular routing of synaptic proteins versus proteins of the noninnervated membrane. Rapsyn being tightly associated with the innervated membrane, it is therefore unlikely that the presence of rapsyn in PGVs results from its redistribution during the purification procedures. The observa- tion that rapsyn was consistently detected at low levels in VR, whereas V43 usually contained both AChR and rapsyn, is puzzling. This led us to assume that several subpopulations of AChRrich PGVs carrying variable amounts of rapsyn do exist. One could suppose that the epitopes on the cytoplasmic moeity of AChRs, recognized by mabs 111 and 155 used for immunopurification of VR fraction, are masked in the PGVs containing larger amount of rapsyn. Consequently, VRs with a low content of rapsyn were selected. PGVs with increasing rapsyn content would result from a gradient of maturation of AChR-containing PGVs to which rapsyn molecules would bind, after their budding from the trans-Golgi network. The observation that Golgi fractions usually contained a low amount of rapsyn (Fig. 1) does agree with this hypothesis.

The morphological analysis by EM of VR and V43 reveals that these fractions correspond to uncoated vesicles (Fig. 2A,B). These vesicles have a diameter of $100 \pm 20 \mathrm{~nm}$, a size similar to that of the PGVs previously characterized (Camus et al., 1998). Immunogold labeling of these PGVs was undertaken to confirm that AChR and rapsyn were indeed comprised in the same vesicles. Approximately $25 \%$ of VRs were labeled with antirapsyn antibodies, a value consistent with the low content of rapsyn in VR. Conversely, a large proportion $(>50 \%)$ of V43s were labeled with anti-AChR antibodies (Fig. $2 C$ ), this confirming that rapsyn and $\mathrm{AChR}$ are present together in the same vesicles. However, the immunogold labeling of AChR in V43 concerns roughly half of the vesicles, a value lower than that expected given the rapsyn/AChR ratio biochemically detected in these vesicles. The underestimation of AChR content in these vesicles may be accounted for, as suggested above, by the partial masking of epitopes corresponding to mAbs 111 and 155 at the cytoplasmic domain of AChR caused by the presence of rapsyn.

\section{Biochemical characterization of rapsyn and AChR in PGVs}

In purified AChR-rich membranes, cross-linking experiments using the heterobifunctional cross-linking reagent SMPB have shown a close proximity between the AChR $\beta$-subunit and rapsyn, suggesting a direct interaction between these two proteins (Burden et al., 1983). We have used this cross-linking procedure to investigate the possible interaction between AChR and rapsyn in PGVs. At variance with our observation on purified AChRrich plasma membranes, we did not observe any cross-link product between rapsyn and AChR in PGVs (data not shown). In similar experiments with crude Torpedo plasma membranes, we did not obtain any cross-linking between AChR and rapsyn, indicating that the detection of a cross-link product, even in the case of the plasma membrane, depends on unknown factors, such as the presence of contaminating membranes, the relative concentration of cross-linking agent, etc. No conclusion can be drawn from these experiments concerning the interaction between AChR and rapsyn in PGVs. However, the partial masking of AChR by rapsyn provided by the immunopurification experiments (see above), suggests that these two proteins are in close proximity in PGVs.

Given the critical role of protein phosphorylation in membrane-cytoskeleton interactions and, in particular, in rapsyndependent aggregation of AChRs triggered by agrin, a nervederived extracellular matrix glycoprotein, we have investigated the phosphorylation of rapsyn during its intracellular trafficking. This was achieved by Western blotting experiments using antiphosphoserine antibodies. In postsynaptic membrane, rapsyn is 

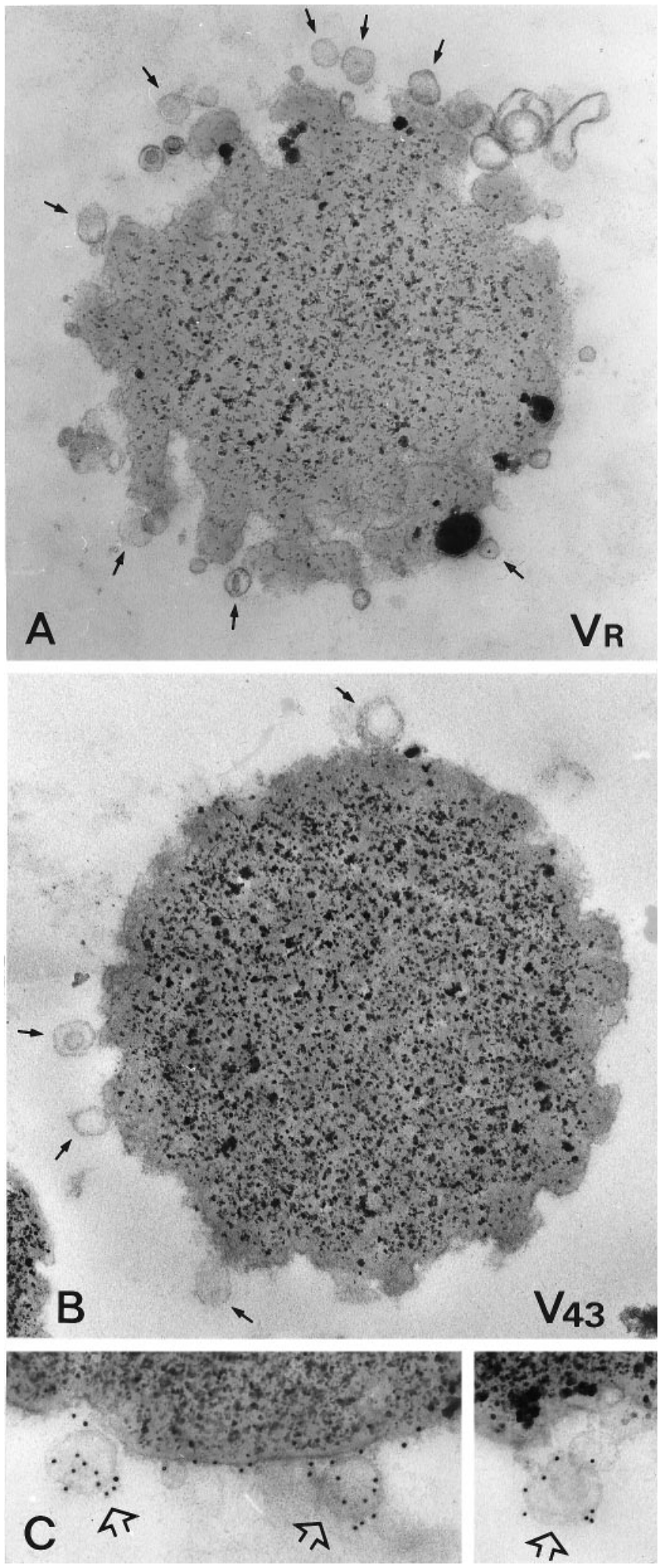

Figure 2. EM analysis and immunogold labeling of VR and V43. After immunopurification on magnetic beads, VR and V43 were directly processed for EM without desorption of the vesicles. VR $(A)$ and V43 $(B)$ vesicles (arrows) appeared uncoated and had a relatively homogeneous size $(100 \pm 20 \mathrm{~nm}$, mean diameter $\pm \mathrm{SD})$. Immunogold labeling of V43 with anti-AChR antibodies $(C)$ disclosed that most of V43 contains AChR. These PGVs thus contain both proteins. A, B, 35,000×; $C$, $50,000 \times$.

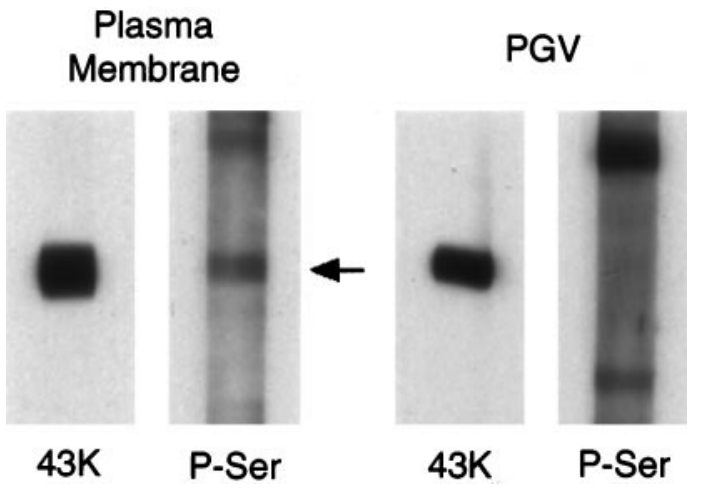

Figure 3. Analysis of rapsyn serine phosphorylation in the exocytic pathway. Serine phosphorylation of rapsyn in plasma membrane and PGV fractions was detected by Western blotting using monoclonal anti-rapsyn and anti-phosphoserine antibodies. At variance with postsynaptic membrane in which rapsyn was phosphorylated on serine residues (arrow), rapsyn in PGV was not phosphorylated. Phosphoserine immunodetection in PGVs was overexposed to ascertain that no band was detected in the 43 $\mathrm{kDa}$ region of the blot.

phosphorylated on serine residues, as previously reported $(\mathrm{Hu}-$ ganir et al., 1984; Hill et al., 1991, but see Balasubramanian and Huganir, 1999). In PGVs, phosphorylation of rapsyn on serine residues was not detected (Fig. 3). This result, taken together with our previous data on AChR phosphorylation showing that the $\beta$ and $\delta$ subunits of AChR in PGVs are not phosphorylated on tyrosine residues (Camus et al., 1998), indicates that the phosphorylation of rapsyn on serine residues and AChR on tyrosine residues specifically occurs at the plasma membrane, likely as a result of innervation (Camus et al., 1999).

\section{In situ localization of rapsyn/AChR carriers vesicles}

In a previous study, we have followed the vesicular trafficking of AChR in the electrocyte by immunogold labeling. Here, we examined the localization of rapsyn in the exocytic pathway. Immunogold labeling experiments were performed on electric tissue fixed after stabilization of the microtubular network. Previous work from our laboratory provided evidence that in these experimental conditions, AChR carriers were observed in the subneural cytoplasm, close to the troughs of the postsynaptic membrane (Camus et al., 1998). Electron microscopy analysis of immunogold-labeled sections using rapsyn antibody reveals that rapsyn immunoreactivity is associated not only with the postsynaptic membrane but also with uncoated vesicles (Fig. 4), sometimes associated with microtubules (Fig. 4B,C). Double-labeling experiment demonstrated the colocalization of AChR and rapsyn in these vesicles (Fig. 4D-F). These observations support the notion that rapsyn initially associates with AChR transport vesicles during their intracellular routing and that the two proteins are subsequently cotargeted to the postsynaptic membrane. Further evidence suggests the contribution of the microtubular network in the targeting of $\mathrm{AChR} / \mathrm{rapsyn}$ transport vesicles to the postsynaptic membrane (Bignami et al., 1998).

\section{DISCUSSION}

Several lines of evidence indicate that rapsyn is critically involved in the clustering of $\mathrm{AChR}$ at the neuromuscular junction. Rapsyn can induce the clustering of $\mathrm{AChR}$ when the two proteins are coexpressed in heterologous cell systems (Froehner et al., 1990; Phillips et al., 1991a; Yu and Hall, 1994; Ramarao and Cohen, 1998). Rapsyn gene invalidation in mice resulted in failure to 

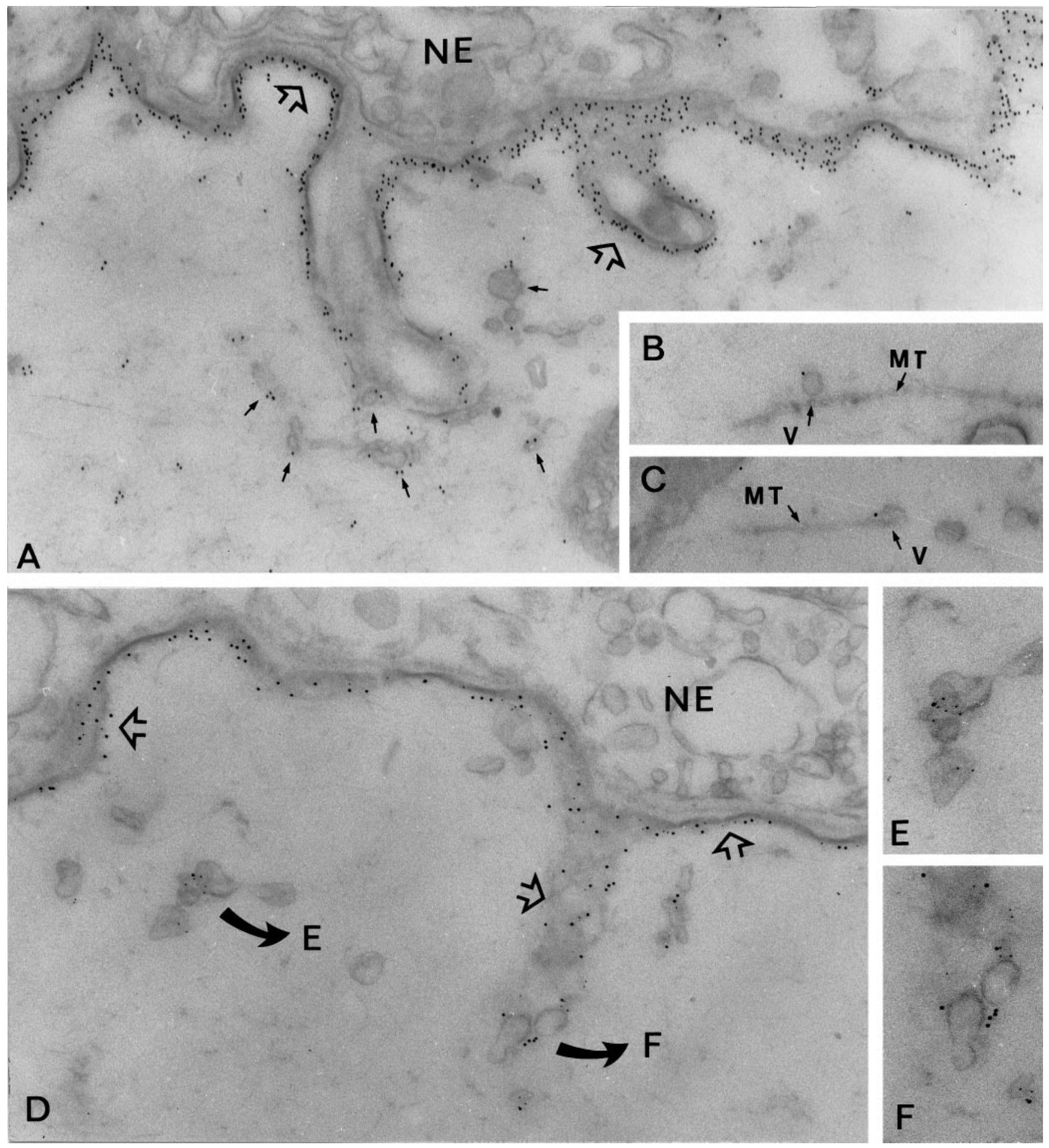

Figure 4. In situ localization of rapsyn and AChR carriers in Torpedo electrocytes at the EM level. These experiments were performed in Torpedo electrocytes after fixation at room temperature and in the presence of Taxol to preserve the microtubular network. $A$, Immunolabeling of rapsyn ( $12 \mathrm{~nm}$ gold particles) was associated with the postsynaptic membrane (open arrows) and was also found in association with numerous uncoated vesicles accumulated close to the innervated membrane folds (arrows). Occasionally, some vesicles were observed in association with microtubules $(B, C)$. $D$, Simultaneous detection of rapsyn and AChR in vesicular carriers was achieved using second antibodies conjugated to 12 or $6 \mathrm{~nm}$ gold particles, respectively. Colocalization of rapsyn and $\mathrm{AChR}$ was obvious in the postsynaptic membrane (open arrows) and was also observed in cytoplasmic vesicles (details in $E$ and $F$ ). $N E$, Nerve ending; $M T$, microtubule; $V$, vesicle. $A, D, 40,000 \times ; B, C, 50,000 \times ; E, F, 55,000 \times$.

cluster AChR and other postsynaptic components at the neuromuscular junction (Gautam et al., 1995). Analysis of these mice further disclosed that rapsyn is involved in the agrin signaling pathway downstream of MuSK, the muscle-specific receptor ty- rosine kinase at the synapse. There, rapsyn acts probably as a relay in the cascade of tyrosine phosphorylation ultimately leading to AChR phosphorylation and clustering (Gillepsie et al., 1996; Apel et al., 1997). Its strict colocalization and its 1:1 stoi- 
chiometry with AChR further suggest that the two proteins are associated in the postsynaptic membrane. However, little is known about their interaction in the process of AChR clustering in the postsynaptic membrane. The current hypothesis is that soluble rapsyn associates with AChRs at the plasma membrane. Recent studies showed, however, that some acylated proteins associate initially with membranes in the exocytic pathway before subsequent targeting to the cell surface. Then, it is worth understanding the membrane targeting of rapsyn compared to that of AChR.

In a recent work, we have shown that the newly synthetized AChRs are directly delivered to the postsynaptic membrane by a mechanism involving intracellular sorting, probably within the Golgi apparatus, followed by a vesicular transport to the postsynaptic membrane (Camus et al., 1998). In the present work, we show that AChR and rapsyn are found in the same ratio in the crude post-Golgi vesicles as in the postsynaptic membrane. This led us to postulate that these two molecules might be associated early in the exocytic pathway and share the same vesicular carriers en route to the postsynaptic membrane. Isolation and characterization of PGVs containing both $\mathrm{AChR}$ and rapsyn and lacking in $\mathrm{Na}, \mathrm{K}$-ATPase indeed confirm that these proteins are cotransported in a distinct population of post-Golgi vesicles.

Our data also point to the existence of several subpopulations of AChR-rich vesicles containing variable amounts of rapsyn. Golgi fractions purified from Torpedo electrocytes usually displayed a low rapsyn content, indicating a possible association of rapsyn at an early step in the exocytic pathway. Alternatively, PGV subpopulations containing variable amounts of rapsyn may result from a gradient of maturation of AChR-containing PGVs to which rapsyn molecules bind, after their budding from the trans-Golgi network. This could be accounted for by coat proteins necessary for the budding of PGVs from the trans-Golgi network, assuming that the association of rapsyn at the cytoplasmic surface occurs more efficiently after uncoating.

These observations are in agreement with recent findings showing that some acylated proteins associate initially with membranes in the exocytic pathway, before their subsequent targeting to the plasma membrane. The acylation of the Src-related tyrosine kinase p56 ${ }^{\text {Lck }}$ (Bijlmakers and Marsh, 1999), of the neurospecific calmodulin-binding protein growth-associated protein 43 (GAP43) (Liu et al., 1994) and of the soluble $N$-ethylmaleimide-sensitive factor attachment protein receptor (SNARE) protein synaptosome-associated protein (SNAP25) (Gonzalo and Linder, 1998), is required not only for their association with subcellular compartments but also for targeting to their proper destination. Moreover, the targeting and/or palmitoylation of these proteins to the cell surface is prevented by brefeldin A, indicating that intracellular trafficking of these acylated proteins depends on a functional secretory pathway (Liu et al., 1994; Gonzalo and Linder, 1998; Bijlmakers and Marsh, 1999). It has further been proposed that palmitoylation, which occurs early in the exocytic pathway, is a sorting signal involved in the specific association of GAP43 and SNAP25 with rapid transport vesicles after budding from trans-Golgi network (Liu et al., 1994; Gonzalo and Linder, 1998). Until now, there is no evidence that myristoylation contributes to the specific association of proteins with transport vesicles, but the present study suggests such a contribution of rapsyn myristoylation in its own targeting in the exocytic pathway. This hypothesis is strengthened by the observation that targeting of rapsyn to the cell surface occurs normally when the $\mathrm{N}$-terminal site of myristoylation is replaced by the site of palmitoylation of GAP43 (Ramarao and Cohen, 1998). The role of brefeldin A in the intracellular trafficking of rapsyn in transfected cells is presently studied in our laboratory.

In vitro experiments have shown that purified Torpedo rapsyn binds tightly to pure liposomes of various composition (Porter and Froehner, 1985). This interaction with lipids cannot, however, explain the coextensive distribution of rapsyn with AChR in situ. Indeed, our observations show that rapsyn does not associate with Na,K-ATPase-containing PGVs (VA), indicating that a specific step is required for the proper targeting of rapsyn to AChRcontaining PGVs. The specific association of rapsyn with AChRtransport vesicles raises the hypothesis of their interaction during vesicular transport. Our investigation of the topography of rapsyn versus AChR using a cross-linking experiment did not succeed in demonstrating a close proximity between these two partners either in crude PGVs or crude plasma membranes, as opposed to purified AChR-rich membranes. However, the failure to detect any cross-link product should not be taken for an absence of interaction between AChR and rapsyn. More favorable conditions for cross-linking experiments, such as with immunopurified vesicles (V43), would be worth testing. Such experiments are unfortunately unrealistic because they require prohibitive amounts of material.

After nerve signaling by agrin, tyrosine phosphorylation plays
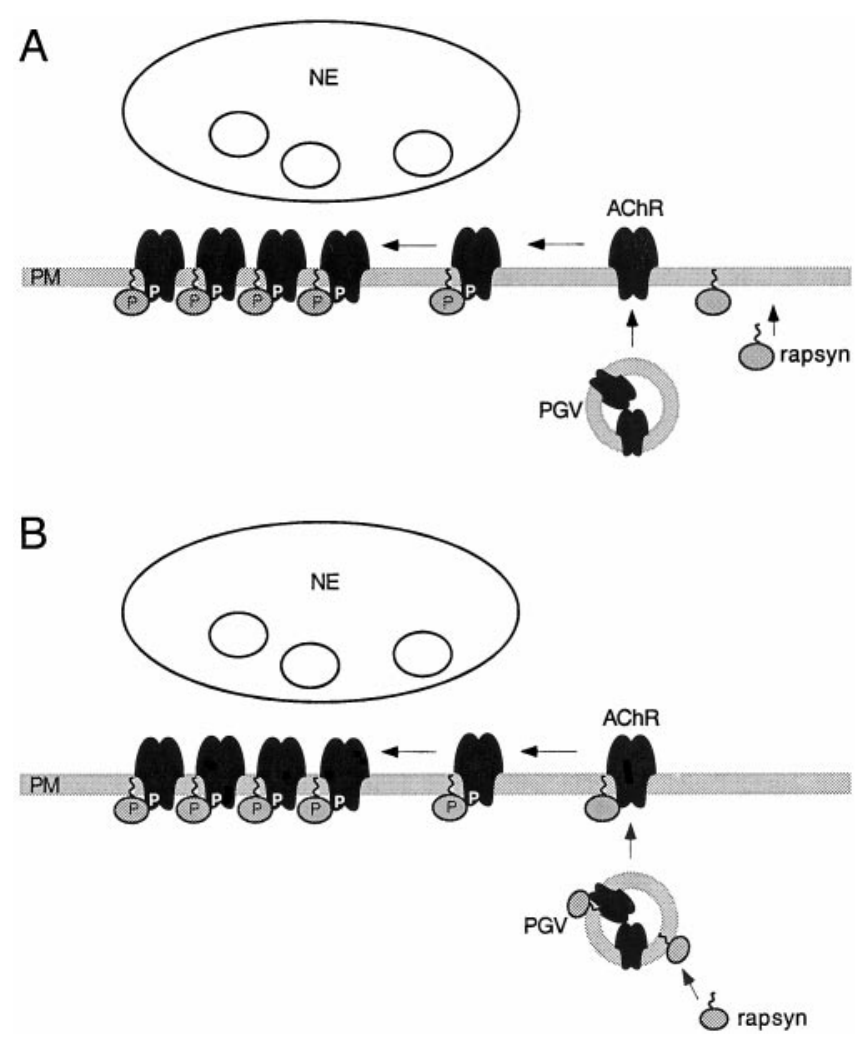

Figure 5. Models of targeting pathways for rapsyn and AChR to the postsynaptic membrane. After synthesis and acylation in the cytoplasm, rapsyn may take two alternative ways to become targeted to the synapse. Either rapsyn is directly inserted in the postsynaptic membrane independently of AChR, targeted via vesicular transporters $(A)$, or rapsyn is initially recruited in the exocytic pathway and subsequently cotargeted with $\mathrm{AChR}(B)$, as suggested in our present work. In $B$, AChR and rapsyn may already associate in vesicles. In both models, rapsyn is inserted selectively in AChR-containing membranes. Tyrosine phosphorylation of the AChRs and serine phosphorylation of rapsyn occurring in the postsynaptic membrane are indicated. 
a critical role in the localization of the AChR at the synapse. The AChR from adult Torpedo is highly tyrosine-phosphorylated on its $\beta$ and $\delta$-subunits (Huganir et al., 1984; Hopfield et al., 1988), and phosphotyrosine immunoreactivity colocalizes with AChR at the mature fish, avian, and mammalian neuromuscular junctions (Qu et al., 1990; Qu and Huganir, 1994; Camus et al., 1999). Tyrosine phosphorylation of the $\beta$-subunit correlates with AChR clustering in agrin-induced myotubes (Wallace et al., 1991; Qu and Huganir, 1994; Meier et al., 1995). Furthermore, treatment of the myotubes with tyrosine protein kinase inhibitors prevents both spontaneous and agrin-induced AChR clustering (Wallace, 1994, 1995; Ferns et al., 1996). The analysis of serine phosphorylation of rapsyn in PGVs show that it is not phosphorylated in exocytic compartments, rapsyn becoming phosphorylated only after its insertion in the postsynaptic membrane. Interestingly, this latter observation is coherent with our previous report on the absence of tyrosine phosphorylation of the AChR $\beta$-subunit in exocytic compartments (Camus et al., 1998). Protein phosphorylation is known to modulate interactions between membrane components and the cytoskeleton, in particular tyrosine phosphorylation is believed to immobilize AChR in postsynaptic membrane probably via interactions with the cytoskeleton (Wallace, 1995). Considering the fact that rapsyn and AChR are found simultaneously in PGVs and are not phosphorylated before their integration in the plasma membrane, we hypothesize that AChR and rapsyn phosphorylation are involved in the regulation of their clustering in the postsynaptic membrane (Fig. 5). In this model, we propose that the AChR/rapsyn pair is first targeted to the plasma membrane and that protein phosphorylation regulates its assembly within the postsynaptic complex. Immunolocalization experiments of phosphorylated AChR in adult Torpedo electrocyte disclosed a mosaic structure with phosphorylated AChRs localized in the postsynaptic membrane just beneath the nerve terminal, and nonphosphorylated AChRs in the troughs of the postsynaptic membrane (Camus et al., 1999). Conversely, rapsyn is present throughout the postsynaptic membrane. These observations are consistent with the notion that post-Golgi vesicles preferentially dock and fuse at the tip of the troughs (Jasmin et al., 1991) of the postsynaptic membrane. In these regions, nonphosphorylated AChR and rapsyn colocalize, whereas AChR phosphorylation occurs in the juxtaneural regions of the postsynaptic membrane, probably as a result from nerve signaling.

The clustering and stabilization of neurotransmitter receptors at synaptic sites share common features: the involvement of extrinsic proteins that interact with cytoplasmic loops of the receptors: rapsyn for $\mathrm{AChR}$, gephyrin for glycine, and GABAA receptors (Meyer et al., 1995; Essrich, 1998), and PDZ domaincontaining proteins PSD-95, GRIP, and Homer for NMDA, AMPA, and metabotropic glutamate receptor, respectively (for review, see Sheng, 1997; Colledge and Froehner, 1998). These proteins are believed to mediate the anchoring of their companion receptor to postsynaptic membrane via an interaction with the cytoskeleton. Our present work suggests an additional dynamic role of this class of proteins in the targeting of neurotransmitter receptors along the biosynthetic pathway.

\section{REFERENCES}

Antony C, Huchet M, Changeux JP, Cartaud J (1995) Developmental regulation of membrane traffic organization during synaptogenesis in mouse diaphragm muscle. J Cell Biol 130:959-968.

Apel ED, Glass DJ, Moscoso LM, Yancopoulos GD, Sanes JR (1997) Rapsyn is required for MuSK signalling and recruits synaptic components to a MuSK-containing scaffold. Neuron 18:623-635.
Balasubramanian S, Huganir RL (1999) Characterization of phosphotyrosine containing proteins at the cholinergic synapse. FEBS Lett 446:95-105.

Bignami F, Camus G, Marchand S, Bailly L, Stetzkowski-Marden F, Cartaud J (1998) Targeting of acetylcholine receptor and $43 \mathrm{kDa}$ rapsyn to the postsynaptic membrane in Torpedo marmorata electrocyte. J Physiol (Paris) 92:177-181.

Bijlmakers MJ JE, Marsh M (1999) Trafficking of an acylated cytosolic protein: newly synthetized $\mathrm{p} 56^{\mathrm{lck}}$ travels to the plasma membrane via the exocytic pathway. J Cell Biol 145:457-468.

Burden SJ, De Palma RL, Gottesman GS (1983) Crosslinking of proteins in acetylcholine receptor-rich membranes: association between the $\beta$-subunit and the $43 \mathrm{kDa}$ subsynaptic protein. Cell 35:687-692.

Camus G, Jasmin BJ, Cartaud J (1998) Polarized sorting of nicotinic acetylcholine receptors to the postsynaptic membrane in Torpedo electrocyte. Eur J Neurosci 10:839-852.

Camus G, Ludosky MA, Bignami F, Marchand S, Cartaud J, Cartaud A (1999) Developmental regulation of tyrosine phosphorylation of the nicotinic acetylcholine receptor in Torpedo electrocyte. Mol Cell Neurosci 13:69-78.

Cartaud J, Changeux JP (1993) Post-transcriptional compartmentalization of acetylcholine receptor biosynthesis in the subneural domain of muscle and electrocyte junctions. Eur J Neurosci 5:191-202.

Cartaud A, Stetzkowski-Marden F, Cartaud J (1993) Identification of dystrophin-binding proteins in membranes from Torpedo electrocyte and rat muscle. J Biol Chem 268:13019-13022.

Colledge M, Froehner SC (1998) To muster a cluster: anchoring neurotransmitter receptors at synapses. Proc Natl Acad Sci USA 95:3341-3343.

Essrich C (1998) Postsynaptic clustering of major GABAA receptor subtypes requires the gamma 2 subunit and gephyrin. Nat Neurosci 1:563-571.

Ferns M, Deiner M, Hall Z (1996) Agrin-induced acetylcholine receptor clustering in mammalian muscle requires tyrosine phosphorylation. J Biol Chem 132:937-944.

Froehner SC, Luetj CW, Scotland PB, Patrick J (1990) The postsynaptic $43 \mathrm{~K}$ protein clusters muscle nicotinic acetylcholine receptors in Xenopus oocytes. Neuron 5:403-410.

Gautam M, Noakes PG, Mudd J, Nichol M, Chu GC, Sanes JR, Merlie JP (1995) Failure of postsynaptic specialization to develop at neuromuscular junctions of rapsyn-deficient mice. Nature 377:232-236.

Gillepsie SKH, Balasubramanian S, Fung ET, Huganir RL (1996) Rapsyn clusters and activates the synapse-specific receptor tyrosine kinase MuSK. Neuron 16:953-962.

Gonzalo S, Linder ME (1998) SNAP-25 palmitoylation and plasma membrane targeting require a functional secretory pathway. Mol Biol Cell 9:585-597.

Griffiths G, Simons K (1986) The trans Golgi network: sorting at the exit site of the Golgi complex. Science 234:438-443.

Hill Jr JA, Nghiêm HO, Changeux JP (1991) Serine-specific phosphorylation of nicotinic receptor associated $43 \mathrm{kDa}$ protein. Biochemistry 30:5579-5585.

Hopfield JF, Tank DW, Greengard P, Huganir RL (1988) Functionnal modulation of the nicotinic acetylcholine receptor by tyrosine phosphorylation. Nature 336:677-680.

Huganir RL, Miles K, Greengard P (1984) Phosphorylation of the nicotinic acetylcholine receptor by an endogenous tyrosine-specific protein kinase. Proc Natl Acad Sci USA 81:6968-6972.

Jasmin BJ, Cartaud J, Bornens M, Changeux JP (1989) Golgi apparatus in chick skeletal muscle: changes in its distribution during end plate development and after denervation. Proc Natl Acad Sci USA 86:7218-7222.

Jasmin BJ, Changeux JP, Cartaud J (1990) Compartmentalization of cold-stable and acetylated microtubules in the subsynaptic domain of chick skeletal muscle fiber. Nature 344:673-675.

Jasmin BJ, Changeux JP, Cartaud J (1991) Organization and dynamics of microtubules in Torpedo marmorata electrocyte: selective association with specialized domains of the postsynaptic membrane. Neuroscience 43:151-162.

Jasmin BJ, Antony C, Changeux JP, Cartaud J (1995) Nerve dependent plasticity of the Golgi complex in skeletal muscle fibers: compartmentalization within the subneural sarcoplasm. Eur J Neurosci 7:470-479.

Kordeli E, Cartaud J, Nghiêm HO, Pradel LA, Dubreuil C, Paulin D, Changeux JP (1986) Evidence for a polarity in the distribution of proteins from the cytoskeleton in Torpedo marmorata electrocytes. J Cell Biol 102:748-761. 
Laemmli UK (1970) Cleavage of structural proteins during the assembly of the head of bacteriophage T4. Nature 227:680-685.

LaRochelle WJ, Froehner SC (1986) Determination of the tissue distributions and relative concentrations of the postsynaptic $43 \mathrm{kDa}$ protein and the acetylcholine receptor in Torpedo. J Biol Chem 261:5270-5274.

Liu Y, Fischer DA, Storm DR (1994) Intracellular sorting of neuromodulin (GAP-43) mutants modified in the membrane targeting domains. J Neurosci 14:5807-5817.

Meier T, Perez GM, Wallace BG (1995) Immobilization of nicotinic acetylcholine receptors in mouse $\mathrm{C} 2$ myotubes by agrin-induced protein tyrosine phosphorylation. J Cell Biol 131:441-451.

Meyer G, Kirsh J, Betz H, Langosch D (1995) Identification of a gephyrin binding motif on the glycine receptor beta subunit. Neuron 15:563-572.

Musil LS, Carr C, Cohen JB, Merlie JP (1988) Acetylcholine receptorassociated $43 \mathrm{~K}$ protein contains covalently bound myristate. J Cell Biol 107:1113-1121.

Peng HB, Froehner SC (1985) Association of the postsynaptic 43K protein with newly formed acetylcholine receptor clusters in cultured muscle cells. J Cell Biol 100:1698-1705.

Phillips WD, Kopta C, Blount P, Gardner PD, Steinbach JH, Merlie JP (1991a) ACh receptor-rich domains organized in fibroblasts by recombinant 43-kilodalton protein. Science 251:568-570.

Phillips WD, Maimone MM, Merlie JP (1991b) Mutagenesis of the 43 $\mathrm{kD}$ postsynaptic protein defines domains involved in plasma membrane targeting and AChR clustering. J Cell Biol 115:1713-1723.

Porter S, Froehner SC (1985) Interaction of the 43K protein with components of Torpedo postsynaptic membranes. Biochemistry 24:425-432.

Qu Z, Huganir RL (1994) Comparison of innervation and agrin-induced protein tyrosine phosphorylation of the nicotinic acetylcholine receptor. J Neurosci 14:6834-6841.

Qu Z, Moritz E, Huganir RL (1990) Regulation of tyrosine phosphorylation of the nicotinic acetylcholine receptor at the rat neuromuscular junction. Neuron 2:367-378.

Ramarao MK, Cohen JB (1998) Mechanism of nicotinic acetylcholine receptor cluster formation by rapsyn. Proc Natl Acad Sci USA 95:4007-4012.

Saitoh T, Changeux JP (1980) Phosphorylation in vitro of membrane fragments from Torpedo marmorata electric organ. Eur J Biochem 105:51-62.

Sanes JR, Lichtman JW (1999) Development of the vertebrate neuromuscular junction. Annu Rev Neurosci 22:389-442.

Schiff PB, Fant J, Horwitz SB (1979) Promotion of microtubule assembly in vitro by Taxol. Nature 277:665-667.

Sheng M (1997) Glutamate receptors put in their place. Nature 386:221-223.

Towbin H, Staehelin T, Gordon J (1979) Electrophoresis transfer of proteins from polyacrylamide gels to nitrocellulose sheets: procedure and some applications. Proc Natl Acad Sci USA 76:4350-4354.

Tzartos SJ, Lindstrom JM (1980) Monoclonal antibodies used to probe acetylcholine receptor structure: localization of the main immunogenic region and detection of similarities between subunits. Proc Natl Acad Sci USA 77:755-759.

Wallace BG (1994) Staurosporine inhibits agrin-induced acetylcholine receptor phosphorylation and aggregation. J Cell Biol 125:661-668.

Wallace BG (1995) Regulation of the interaction of nicotinic acetylcholine receptors with the cytoskeleton by agrin-activated protein tyrosine kinase. J Cell Biol 128:1121-1129.

Wallace BG, Qu Z, Huganir RL (1991) Agrin induces phosphorylation of the nicotinic acetylcholine receptor. Neuron 6:869-878.

Wandinger-Ness A, Bennett MK, Antony C, Simons K (1990) Distinct transport vesicles mediate the delivery of plasma membrane proteins to the apical and basolateral domains of MDCK cells. J Cell Biol 111:987-1000.

Wilcox C, Hu JS, Olson EN (1987) Acylation of proteins with myristic acid occurs cotranslationally. Science 238:1275-1278.

Yu XM, Hall ZW (1994) The role of the cytoplasmic domains of individual subunits of the acetylcholine receptor in $43 \mathrm{kDa}$ protein-induced clustering in COS cells. J Neurosci 14:785-795. 